\title{
A Weaker Constraint Qualification of Globally Convergent Homotopy Method for a Multiobjective Programming Problem
}

\author{
Guangming Yao ${ }^{1}$, Wen Song ${ }^{2}$ \\ ${ }^{1}$ Department of Mathematics, Clarkson University, Potsdam, USA \\ ${ }^{2}$ Department of Mathematics, Harbin Normal University, Harbin, China \\ Email: gyao@clarkson.edu,wsong218@yahoo.com.cn
}

Received September 29, 2012; revised January 9, 2013; accepted January 16, 2013

\begin{abstract}
In this paper, we prove that the combined homotopy interior point method for a multiobjective programming problem introduced in Ref. [1] remains valid under a weaker constrained qualification - the Mangasarian-Fromovitz constrained qualification, instead of linear independence constraint qualification. The algorithm generated by this method associated to the Karush-Kuhn-Tucker points of the multiobjective programming problem is proved to be globally convergent.
\end{abstract}

Keywords: Multiobjective Programming Problem; Homotopy Method; KKT Condition; Efficient Solution; MFCQ

\section{Introduction}

Let $\mathbb{R}^{n}$ be the $n$-dimensional Euclidean space, and let $\mathbb{R}_{+}^{n}$ and $\mathbb{R}_{++}^{n}$ denote the nonnegative and positive $\mathbb{R}^{n}$, respectively. For any two vectors $y=\left(y_{1}, y_{2}, \cdots, y_{n}\right)$ and $z=\left(z_{1}, z_{2}, \cdots, z_{n}\right)$ in $\mathbb{R}^{n}$, we use the following conventions: $y=z$ iff $y_{i}=z_{i}, i=1,2, \cdots, n$. Similarly, we can define $y \leqq z, y<z$, and $y \leq z$.

Consider the following multiobjective programming problem (MOP)

where

$$
\begin{aligned}
& \min f(x) \\
& \text { s.t. } g(x) \leqq 0, h(x)=0,
\end{aligned}
$$

$$
\begin{aligned}
& x \in \mathbb{R}^{n}, f=\left(f_{1}, f_{2}, \cdots, f_{p}\right)^{\mathrm{T}}, \\
& g=\left(g_{1}, g_{2}, \cdots, g_{m}\right)^{\mathrm{T}}, h=\left(h_{1}, h_{2}, \cdots, h_{s}\right)^{\mathrm{T}} .
\end{aligned}
$$

We assume that all $f_{i}, i=1, \cdots, p, g_{j}, j \in I$ and $h_{k}, k \in J$ are twice continuously differentiable functions, where $I=\{1,2, \cdots, m\}, J=\{1,2, \cdots, s\}$.

Let

$$
\begin{aligned}
& \Omega=\left\{x \in \mathbb{R}^{n}: g(x)<0, h(x)=0\right\}, \\
& \Omega_{1}=\left\{x \in \mathbb{R}^{n}: g(x)<0\right\}, \\
& \Lambda^{++}=\left\{\lambda \in \mathbb{R}_{++}^{p}: \sum_{i=1}^{p} \lambda_{i}=1\right\}, \\
& I(x):=\left\{j \in I: g_{j}(x)=0\right\} .
\end{aligned}
$$

It is well known that if $x$ is an efficient solution of
(MOP), under some constraint qualifications, such as the Kuhn and Tucker constraint qualification (see Ref. [2]) or the Abadie constraint qualification (see Ref. [3]), then the following Karush-Kuhn-Tucker (KKT) condition at $x$ for (MOP) holds (see Refs. [4,5]):

$$
\left\{\begin{array}{l}
\nabla f(x)^{\mathrm{T}} \lambda+\nabla g(x)^{\mathrm{T}} u+\nabla h(x)^{\mathrm{T}} v=0 \\
U g(x)=0 \\
h(x)=0
\end{array}\right.
$$

where $\lambda \in \mathbb{R}_{+}^{p} \backslash\{0\}, u \in \mathbb{R}_{+}^{m}, v \in \mathbb{R}^{s}$, and

$$
U=\operatorname{diag}\left\{u_{1}, u_{2}, \cdots, u_{m}\right\} .
$$

We say that $x$ is a KKT point of (MOP) if it satisfies the KKT condition.

Since the remarkable papers of Kellogg et al. (Ref. [6]) and Chow et al.(Ref. [7]) have been published, more and more attention has been paid to the homotopy method. As a globally convergent method, the homotopy method (or path-following method) now becomes an important tool for numerically solving nonlinear problems includeing nonlinear mathematical programming and complementarily problems (see Refs. [3,4]).

In 1988, Megiddo (see Ref. [8]) and Kojima et al. (see Ref. [9]) discovered that the Karmakar interior point method was a kind of path-following method for solving linear programming. Since then, the interior path-following method has been generalized to convex programming, and becomes one of the main methods for solving mathematical programming problems. Among most interior 
methods, one of the main ideas is numerically tracing the center path generated by the optimal solution set of the so-called logarithmic barrier function. Usually, the strict convexity of the logarithmic barrier function or nonemptiness and boundedness of the feasible set (see Ref. [10]) are needed. In 1997, Lin, Yu and Feng (see Ref. [11]) presented a new interior point method-combined homotopy interior point method (CHIP method)-for convex nonlinear programming without such assumptions. Subsequently, Lin, Li and Yu (see Ref. [12]) generalized CHIP method to general nonlinear programming where, instead of convexity condition, they used a more general "normal cone condition".

In 2003, Lin, Zhu and Sheng (see Ref. [13]) generalized CHIP method to convex multiobjective programming(CMOP) with only inequality constraints. Instead of (CMOP), they considered an associated non-convex nonlinear scalar optimization problem and constructed the homotopy mapping.

In Refs. [1,14], we considered a combined homotopy interior point method for the multiobjective programming (MOP) under the condition linearly independent constraint qualification (LICQ). To find a KKT point of (MOP), we construct a homotopy as follows

$$
0=H\left(\omega, \omega^{0}, \mu\right)=\left[\begin{array}{l}
(1-\mu)\left(\nabla f(x)^{\mathrm{T}} \lambda+\nabla g(x)^{\mathrm{T}} u\right)+\nabla h(x)^{\mathrm{T}} v+\mu\left(x-x^{0}\right) \\
h(x) \\
U g(x)-\mu U^{0} g\left(x^{0}\right) \\
(1-\mu)\left(1-\sum_{i=1}^{p} \lambda_{i}\right) e-\mu\left(\lambda^{2 / 5}-\left(\lambda^{0}\right)^{2 / 5}\right)
\end{array}\right]
$$

where $\omega^{0}=\left(x^{0}, \lambda^{0}, u^{0}, v^{0}\right) \in \Omega \times \Lambda^{++} \times R_{++}^{m} \times\{0\}, e=(1,1, \cdots, 1)^{\mathrm{T}} \in R^{p}, \quad \omega=(x, \lambda, u, v) \in \bar{\Omega} \times R_{++}^{p+m} \times R^{s}, \mu \in[0,1]$, $\lambda^{2 / 5}=\left(\lambda_{1}^{2 / 5}, \ldots, \lambda_{p}^{2 / 5}\right)$, and $\left(\lambda^{0}\right)^{2 / 5}=\left(\left(\lambda_{1}^{0}\right)^{2 / 5}, \ldots,\left(\lambda_{p}^{0}\right)^{2 / 5}\right)$.

Let

$$
H_{\omega^{0}}^{-1}(0)=\left\{(\omega, \mu) \in \Omega \times \mathbb{R}_{++}^{p+m} \times \mathbb{R}^{s} \times(0,1]: H\left(\omega, \omega^{0}, \mu\right)=0\right\} .
$$

Let $A \subset \mathbb{R}^{n}$ be a nonempty closed set and $x \in A$. We recall that the Fréchet normal cone of $A$ at $x$ is defined as

$$
N_{A}(x)=\left\{x^{*} \in \mathbb{R}^{n} \mid \limsup _{x^{\prime} \stackrel{A}{\longrightarrow} x} \frac{\left\langle x^{*}, x^{\prime}-x\right\rangle}{\left\|x^{\prime}-x\right\|} \leq 0\right\} .
$$

We used the following basic assumptions which are commonly used in that literature:

(A1) $\Omega$ is nonempty (Slater condition) and bounded;
(A2) (LICQ) $\forall x \in \bar{\Omega}$, the matrix

$$
\left(\nabla h(x)^{\mathrm{T}}, \nabla g_{j}(x)^{\mathrm{T}}: j \in I(x)\right)
$$

is a matrix of full column rank;

(A3) Normal condition:

$$
\forall x \in \bar{\Omega},\left\{x+\hat{N}_{\bar{\Omega}}(x)\right\} \cap \bar{\Omega}=\{x\} .
$$

It is well known that if condition (A2) holds, then

$$
\hat{N}_{\bar{\Omega}}(x)=\left\{\sum_{i \in I(x)} u_{i} \nabla g_{i}(x)+\sum_{j \in J} v_{j} \nabla h_{j}(x): u_{i} \geq 0, i \in I(x), v \in \mathbb{R}^{s}\right\} .
$$

We have proved the following convergence result in Ref. [1].

Theorem 1.1 (Convergence of the method) Suppose $f_{i}, g_{j}$, and $h_{k}$ are twice continuously differentiable functions such that the conditions (A1), (A2), and (A3) hold. Then for almost all

$$
\omega^{0} \in \Omega \times \Lambda^{++} \times R_{++}^{m} \times R^{s},
$$

the zero-point set $H_{\omega^{0}}^{-1}(0)$ of the homotopy map (2) contains a smooth curve $\Gamma_{\omega^{0}} \subset \bar{\Omega} \times R_{+}^{p+m+s} \times(0,1]$, which starts from $\left(\omega^{0}, 1\right)$. As $\mu \rightarrow 0$, the limit set
$T \times\{0\} \subset \bar{\Omega} \times R_{+}^{p+m+s} \times\{0\}$ of $\Gamma_{\omega^{0}}$ is nonempty, and the $x$-component of every point in $T$ is a KKT point of (MOP).

Recently, many researchers extended and improved the results in Ref. [1] to convex multiobjective programming problem, see Ref. [14-17]. The purpose of this paper is to show that Theorem 1.1 remains true under the condition MFCQ instead of LICQ. The paper is organized as following. In Section 2, we prove the existence and convergence of a smooth homotopy path from almost any interior initial point $\omega^{0}$ to a solution of the 
KKT system of (MOP) under the condition MFCQ.

\section{Main Results}

We need the following elementary condition.

$\left(A 2^{\prime}\right)$ (MFCQ) For every $x \in \bar{\Omega}$, the following conditions hold:

- $\nabla h_{j}(x), j \in J$, are linear independent;

- there exists a $p \in R^{n}$ such that

$$
\nabla g_{i}(x) p>0, i \in I(x) \text { and } \nabla h_{j}(x) p=0, j \in J .
$$

Clearly, condition (A2) implies (A2'). It is also known that if $\left(A 2^{\prime}\right)$ holds, then (3) remains valid.

By using an analogue argument as in Ref. [1], we can prove the following two theorems.

Theorem 2.1 Suppose that $\Omega \neq \varnothing$ and conditions (A1), $\left(A 2^{\prime}\right)$ hold. Then for almost all initial points $\omega^{0} \in \Omega \times \Lambda^{++} \times \mathbb{R}_{++}^{m} \times\{0\}, 0$ is a regular value of $H_{\omega^{0}}$ and $H_{\omega^{0}}^{-1}(0)$ consists of some smooth curves. Among them, a smooth curve, say $\Gamma_{\omega^{0}}$ starts from $\left(\omega^{0}, 1\right)$.

Theorem 2.2 Suppose that $\Omega \neq \varnothing$ and conditions (A1), (A2') hold. For a given $\omega^{0} \in \Omega \times \Lambda^{++} \times \mathbb{R}_{++}^{m} \times \mathbb{R}^{s}$, if 0 is a regular value of $H_{\omega^{0}}$, then the projection of the smooth curve $\Gamma_{\omega^{0}}$ on the $\lambda_{\lambda}^{\omega^{0}}$ component is bounded.

We next prove that $\Gamma_{\omega^{0}}$ is a bounded curve.

Theorem 2.3 (Boundedness) Suppose that the conditions (A1), $\left(A 2^{\prime}\right)$, and (A3) hold. Then for a given $\omega^{0} \in \Omega \times \Lambda^{++} \times \mathbb{R}_{++}^{m} \times \mathbb{R}^{s}$, if 0 is a regular value of $H_{\omega^{0}}$, then $\Gamma_{\omega^{0}}$ is a bounded curve.

Proof: By Theorem 2.2, it is sufficient to show that the $(u, v)$ - component of smooth curve is bounded. Suppose that there exists a sequence $\left\{\left(\omega^{k}, \mu_{k}\right)\right\} \subset \Gamma_{\omega^{0}}$, such that

and

$$
x^{k} \rightarrow x^{*}, \mu_{k} \rightarrow \mu_{*}, \lambda^{k} \rightarrow \lambda^{*}
$$

$$
\left\|\left(u^{k}, v^{k}\right)\right\| \rightarrow \infty(k \rightarrow \infty),
$$

where $x^{*} \in \Omega, \mu_{*} \in[0,1], \lambda^{*} \geqq 0$. Since closed unit circle of $R^{m+s}$ is compact, without loss of generality we can assume that

$$
\lim _{k \rightarrow \infty} \frac{\left(u^{k}, v^{k}\right)}{\left\|\left(u^{k}, v^{k}\right)\right\|}=\left(u^{*}, v^{*}\right) .
$$

Clearly, $u^{*} \geqq 0,\left\|\left(u^{*}, v^{*}\right)\right\|=1$. By (2), we have

$$
\begin{gathered}
\left(1-\mu_{k}\right)\left(\nabla f\left(x^{k}\right)^{\mathrm{T}} \lambda^{k}+\nabla g\left(x^{k}\right)^{\mathrm{T}} u^{k}\right) \\
+\nabla h\left(x^{k}\right)^{\mathrm{T}} v^{k}+\mu_{k}\left(x^{k}-x^{0}\right)=0, \\
U^{k} g\left(x^{k}\right)-\mu_{k} U^{0} g\left(x^{0}\right)=0 .
\end{gathered}
$$

Let

$$
\begin{aligned}
& I_{1}\left(x^{*}\right)=\left\{i \in I: \lim _{k \rightarrow \infty} u_{i}^{k}=\infty\right\}, \\
& I_{2}\left(x^{*}\right)=\left\{j \in J: \lim _{k \rightarrow \infty} v_{j}^{k}=\infty\right\} .
\end{aligned}
$$

By (5), we know $I_{1}\left(x^{*}\right) \subset I\left(x^{*}\right)$.

Rewrite (4) as

$$
\begin{aligned}
& \left(1-\mu_{k}\right)\left[\nabla f\left(x^{k}\right)^{\mathrm{T}} \lambda^{k}+\sum_{i \notin I\left(x^{*}\right)} u_{i}^{k} \nabla g_{i}\left(x^{k}\right)\right. \\
& \left.+\sum_{i \in I\left(x^{*}\right)} u_{i}^{k} \nabla g_{i}\left(x^{k}\right)\right]+\nabla h\left(x^{k}\right)^{\mathrm{T}} v^{k}+\mu_{k}\left(x^{k}-x^{0}\right)=0 .
\end{aligned}
$$

Divide (6) by $\left\|\left(u^{k}, v^{k}\right)\right\|$ and let $k \rightarrow \infty$, since $\left\|\left(u^{k}, v^{k}\right)\right\| \rightarrow \infty,(6)$ becomes

$$
\nabla g_{I}\left(x^{*}\right)^{\mathrm{T}}\left(\left(1-\mu_{*}\right) u_{I}^{*}\right)+\nabla h\left(x^{*}\right)^{\mathrm{T}} v^{*}=0,
$$

where

$$
\begin{aligned}
& \nabla g_{I}\left(x^{*}\right)=\left\{\left(g_{i}\left(x^{*}\right): i \in I\left(x^{*}\right)\right)\right\}, \\
& u_{I}^{*}=\left\{\left(u_{i}^{*}: i \in I\left(x^{*}\right)\right)\right\} .
\end{aligned}
$$

1) If $u_{I}^{*}=0$, then $u^{*}=0$, and $\nabla h\left(x^{*}\right)^{\mathrm{T}} v^{*}=0$. By $\left(A 2^{\prime}\right), v^{*}=0$. This is a contradiction with $\left\|\left(u^{*}, v^{*}\right)\right\|=1$.

2) If $u_{I}^{*} \neq 0$, we consider the following two cases:

1. If $\mu_{*} \in[0,1)$, we know $\left(1-\mu^{*}\right) u_{I}^{*} \geq 0$ because of $u_{I}^{*} \neq 0$. By $\left(A 2^{\prime}\right)$, there exists a nonzero vector $p \in R^{n}$, such that

$$
p^{\mathrm{T}} \nabla g_{I}\left(x^{*}\right)^{\mathrm{T}}>0 \text { and } p^{\mathrm{T}} \nabla h\left(x^{*}\right)^{\mathrm{T}}=0 .
$$

This, together with (7), implies that $\left(1-\mu_{*}\right) u_{I}^{*}=0$, which is a contradiction.

2. If $\mu_{*}=1$, by (7) and $\left(A 2^{\prime}\right)$, we know $v^{*}=0$. So,

$$
\left\|u^{*}\right\|=1 \text { since }\left\|\left(u^{*}, v^{*}\right)\right\|=1 \text {. }
$$

Because of (5), $I_{1}\left(x^{*}\right)=I\left(x^{*}\right) \neq \varnothing$. Thus

$$
x^{*} \in \partial \Omega_{1},\left\|u_{i}^{k}\right\| \rightarrow \infty(k \rightarrow \infty), \forall i \in I .
$$

Without loss of generality, we can assume that

$$
u_{I}^{k}>0, \mu_{k} \neq 1(\forall k \in N) \text {, and } \lim _{k \rightarrow \infty} \frac{u_{I}^{k}}{\left\|u_{I}^{k}\right\|}=u \text {. }
$$

Hence $u \geq 0,\|u\|=1$.

a) If $I_{2}\left(x^{*}\right)=\varnothing$, then $\left\{v^{k}\right\}$ is bounded. We may assume $\lim _{k \rightarrow \infty} v^{k}=v$. Divide (6) by $\left\|\left(1-\mu_{k}\right) u_{I}^{k}\right\|$ and let $k \rightarrow \infty$, (6) becomes 


$$
\begin{aligned}
& \nabla g_{I}\left(x^{*}\right)^{\mathrm{T}} u \\
& +\lim _{k \rightarrow \infty}\left[\frac{1}{\left\|\left(1-\mu_{k}\right) u_{I}^{k}\right\|}\left(\nabla h\left(x^{k}\right)^{\mathrm{T}} v^{k}+\mu_{k}\left(x^{k}-x^{0}\right)\right)\right]=0 .
\end{aligned}
$$

This implies that

$$
\lim _{k \rightarrow \infty} \frac{1}{\left\|\left(1-\mu_{k}\right) u_{I}^{k}\right\|}
$$

exists. Indeed,

$$
\begin{aligned}
& \lim _{k \rightarrow \infty}\left[\nabla h\left(x^{k}\right)^{\mathrm{T}} v^{k}+\mu_{k}\left(x^{k}-x^{0}\right)\right] \\
& =\nabla h\left(x^{*}\right)^{\mathrm{T}} v+x^{*}-x^{0} .
\end{aligned}
$$

If

$$
\nabla h\left(x^{*}\right)^{\mathrm{T}} v+x^{*}-x^{0}=0
$$

that is

$$
\nabla h\left(x^{*}\right)^{\mathrm{T}} v+x^{*}=x^{0} .
$$

By condition (A3), $x^{*}=x^{0}$. This is impossible since $x^{*} \in \partial \Omega$. So

$$
\nabla h\left(x^{*}\right)^{\mathrm{T}} v+x^{*}-x^{0} \neq 0 .
$$

By (9), we then have that $\lim _{k \rightarrow \infty} \frac{1}{\left\|\left(1-\mu_{k}\right) u_{I}^{k}\right\|}$ exists. Assume $\lim _{k \rightarrow \infty} \frac{1}{\left\|\left(1-\mu_{k}\right) u_{I}^{k}\right\|}=\mu$.

Then $\mu \geq 0$.

If $\mu>0$, (9) becomes

$$
\nabla g_{I}\left(x^{*}\right)^{\mathrm{T}} \frac{u}{\mu}+\nabla h\left(x^{*}\right)^{\mathrm{T}} v+x^{*}=x^{0} .
$$

This contradicts to condition (A3).

If $\mu=0$, (9) becomes

$$
\nabla g_{I}\left(x^{*}\right)^{\mathrm{T}} u=0 .
$$

By $\left(A 2^{\prime}\right)$, there exists a nonzero vector $p \in R^{n}$ such that

$$
\nabla g_{I}\left(x^{*}\right) p>0, \text { and } p^{\mathrm{T}} \nabla g_{I}\left(x^{*}\right)^{\mathrm{T}} u=0 .
$$

Thus $u=0$, which contradicts $\|u\|=1$.

b) If $I_{2}\left(x^{*}\right) \neq \varnothing$, without loss of generality, we can assume that

$$
\lim _{k \rightarrow \infty} \frac{\left(\left(1-\mu_{k}\right) u_{I}^{k}, v^{k}\right)}{\left\|\left(\left(1-\mu_{k}\right) u_{I}^{k}, v^{k}\right)\right\|}=(\hat{u}, \hat{v}),
$$

where $\|(\hat{u}, \hat{v})\|=1$. Since

$$
\left\|\left(\left(1-\mu_{k}\right) u_{I}^{k}, v^{k}\right)\right\| \rightarrow \infty \text { as } k \rightarrow \infty,
$$

we divide (4) by $\left\|\left(\left(1-\mu_{k}\right) u_{I}^{k}, v^{k}\right)\right\|$, and let $k \rightarrow \infty$, we have that

$$
\nabla g_{I}\left(x^{*}\right)^{\mathrm{T}} \hat{u}+\nabla h\left(x^{*}\right)^{\mathrm{T}} \hat{v}=0 .
$$

If $\hat{u}=0$, then

$$
\nabla h\left(x^{*}\right)^{\mathrm{T}} \hat{v}=0 .
$$

By condition $\left(A 2^{\prime}\right), \hat{v}=0$. This is a contradiction since $\|(\hat{u}, \hat{v})\|=1$.

If $\hat{u} \neq 0$, by $\left(A 2^{\prime}\right)$, there is a nonzero vector $p \in R^{n}$ such that

$$
p^{\mathrm{T}} \nabla g_{I}\left(x^{*}\right)^{\mathrm{T}}>0 \text {, and } p^{\mathrm{T}} \nabla h\left(x^{*}\right)^{\mathrm{T}}=0 .
$$

This, together with (11), implies $\hat{u}=0$. This is a contradiction.

Therefore, $\Gamma_{\omega^{0}}$ is a bounded curve.

By an analogue argument as in Ref. [1], it is easy to show the following result.

Theorem 2.4 (Convergence of the method) Suppose that the conditions (A1), (A2'), and (A3) hold. Then for almost all $\omega^{0} \in \Omega \times \Lambda^{++} \times \mathbb{R}_{++}^{m} \times \mathbb{R}^{s}$, the zero-point set $H_{\omega^{0}}^{-1}(0)$ of the homotopy map (2) contains a smooth curve $\Gamma_{\omega^{0}} \subset \bar{\Omega} \times \mathbb{R}_{+}^{p+m} \times(0,1]$, which starts from $\left(\omega^{0}, 1\right)$. As $\mu \rightarrow 0$, the limit set $T \times\{0\} \subset \bar{\Omega} \times \mathbb{R}_{+}^{p+m} \times\{0\}$ of $\Gamma_{\omega^{0}}$ is nonempty, and every point in $T$ is a solution of (1).

Therefore, Theorem 2.4 shows that for almost all $\omega^{0} \in \Omega \times \mathbb{R}_{++}^{p+m} \times \mathbb{R}^{s} \times\{0\}$, the homotopy Equation (2) generates a smooth curve $\Gamma_{\omega^{0}}$ starts from $\left(\omega^{0}, 1\right)$ which is called the homotopy path, the limit set $T \times\{0\} \subset \bar{\Omega} \times R_{+}^{p+m+s} \times\{0\} \quad$ of $\Gamma_{\omega^{0}}$ is nonempty, and the $x$-component of every point in $T$ is a KKT point of (MOP), the $\omega$-component of the homotopy path is the solution of (1) as $\mu$ goes to 0 .

\section{REFERENCES}

[1] W. Song and G. M. Yao, "Homotopy Method for General Multiobjective Programming Problems," Journal of Optimization Theory and Applications, Vol. 138, No. 1, 2008, pp. 139-153. doi:10.1007/s10957-008-9366-6

[2] H. W. Kuhn and A. W. Tucker, "Nonlinear Programming," Proceedings of the Second Berkeley Symposium on Mathematical Statistics and Probability, University of California Press, Berkeley, 1951.

[3] T. Maeda, "Second-Order Conditions for Efficiency in Nonsmmoth Multiobjective Optimization Problems," Journal of Optimization Theory and Applications, Vol. 122, No. 3, 2004, pp. 521-538. 


\section{doi:10.1023/B:JOTA.0000042594.46637.b4}

[4] C. Y. Lin and J. L. Dong, "Methods and Theories in Multiobjective Optimization," Jinlin Education Press, Changchun, 1992.

[5] M. Abadie, "Generalized Kuhn-Tucker Conditions for Mathematical Programming," SIAM Journal on Control, Vol. 7, No. 2, 1969, pp. 232-241. doi:10.1137/0307016

[6] R. B. Kellogg, T. Y. Li and J. A. Yorke, "A Constructive Proof of the Brouwer Fixed-Point Theorem and Computational Results," SIAM Journal on Numerical Analysis, Vol. 13, No. 4, 1976, pp. 473-483. doi:10.1137/0713041

[7] S. N. Chow, J. Mallet-Paret and J. A. Yorke, "Finding Zeros of Maps: Homotopy Methods That are Constructive with Probability One," Mathematical Computation, Vol. 32, 1978, pp. 887-899. doi:10.1090/S0025-5718-1978-0492046-9

[8] N. Megiddo, "Pathways to the Optimal Set in Linear Programming, in Progress in Mathematical Programming, Interior Point and Related Methods," Springer, New York, 1988, pp. 131-158.

[9] M. Kojima, S. Mizuno and A. Yoshise, "A Primal-Dual Interior Point Algorithm for Linear Programming," In: N. Megiddo, Ed., Progress in Mathematical Programming, Interior Point and Related Methods, Springer, New York, 1988, pp. 29-47.

[10] E. L. Allgower and K. Georg, "Numerical Continuation Methods: An Introduction," Springer Verlag, Berlin, 1990. doi:10.1007/978-3-642-61257-2

[11] Z. H. Lin, B. Yu and G. C. Feng, "A Combined Homotopy Interior Method for Convex Nonlinear Program- ming," Applied Mathematics and Computation, Vol. 84, No. 2-3, 1997, pp. 193-211. doi:10.1016/S0096-3003(96)00086-0

[12] Z. H. Lin, Y. Li and B. Yu, "A Combined Homotopy Interior Point Method for General Nonlinear Programming Problems," Applied Mathematics and Computation, Vol. 80, No. 2-3, 1996, pp. 209-224. doi:10.1016/0096-3003(95)00295-2

[13] Z. H. Lin, D. L. Zhu and Z. P. Sheng, "Finding a Minimal Efficient Solution of a Convex Multiobjective Program," Journal of Optimization Theory and Applications, Vol. 118, No. 1, 2003, pp. 587-600. doi:10.1023/A:1024739508603

[14] Y. F. Shang and B. Yu, "A Constraint Shifting Homotopy Method for Convex Multi-Objective Programming," Journal of Computational and Applied Mathematics, Vol. 236, No. 5, 2011, pp. 640-646.

[15] X. Zhao, S. G. Zhang and Q. H. Liu, "Homotopy InteriorPoint Method for a General Multiobjective Programming Problem," Journal of Applied Mathematics, Vol. 2012, 2012, Article ID: 497345.

[16] N. Kim and L. Thuy, "An Algorithm for Generating Efficient Outcome Points for Convex Multiobjective Programming Problem," Intelligent Information and Database Systems Lecture Notes in Computer Science, Vol. 5991, No. 2010, 2010, pp. 390-399.

[17] Z. Chen, "Multiobjective Optimization Problems, Vector Variational Inequalities and Proximal-Type Methods," Dissertations, Hong Kong Polytechnic University, Kowloon, 2010. 